\title{
Nonlinear-dispersive similariton of passive fiber
}

\section{A. Zeytunyan \\ G. Yesayan \\ L. Mouradian \\ Imouradian@ysu.am}

\section{P. Kockaert}

\section{Ph. Emplit}

\section{F. Louradour}

\section{A. Barthélémy}

\author{
Ultrafast Optics Laboratory, Faculty of Physics, Yerevan State University, 1 Alex Manoogian \\ Street, Yerevan 0025, Armenia \\ Ultrafast Optics Laboratory, Faculty of Physics, Yerevan State University, 1 Alex Manoogian \\ Street, Yerevan 0025, Armenia \\ Ultrafast Optics Laboratory, Faculty of Physics, Yerevan State University, 1 Alex Manoogian \\ Street, Yerevan 0025, Armenia \\ OPERA photonique, Université Libre de Bruxelles, CP 194/5, 50 Avenue F. D. Roosevelt, \\ B-1050 Bruxelles, Belgium \\ OPERA photonique, Université Libre de Bruxelles, CP 194/5, 50 Avenue F. D. Roosevelt, \\ B-1050 Bruxelles, Belgium \\ XLIM Institut de Recherche, Département Photonique, 123 Avenue A. Thomas, F-87060 \\ Limoges, France \\ XLIM Institut de Recherche, Département Photonique, 123 Avenue A. Thomas, F-87060 \\ Limoges, France
}

Using the spectral-interferometry method for the short pulse complete characterization, we demonstrate the nature and distinctive properties of the similariton generated in single-mode fiber without gain (passive fiber) due to the combined impacts of nonlinearity and dispersion. The nonlinear-spectronic character of such a similariton, with the key specificity of linear chirping, leads to its self-spectrotemporal imaging, important for applications to the signal analysis - synthesis problems in ultrafast optics. [D0I: 10.2971/je0S.2009.09009]

Keywords: fiber, femtosecond, chirp, spectral interferometry, spectron, similariton, spectrotemporal similarity and imaging

\section{INTRODUCTION}

In the last decade self-similarity in optics, parabolic pulses and similaritons along with the soliton optics attract the attention of many researchers due to the academic interest and prospects of applications in ultrafast optics [1]. In the beginning of the 90's Anderson and co-authors predicted a parabolic solution for the nonlinear Schrödinger equation with dispersion and high nonlinearity, that is, the self-similar propagation of a pulse with parabolic temporal, spectral and phase profiles [2]. In practice the generation of such kind of pulses is possible in active fibers such as rare-earth-doped fiber amplifiers [3], Raman fiber amplifiers [4], and also in fibers with distributed dispersion [5]. Such a similariton can be generated in a laser resonator [6] as well, or in a tapered fiber with decreasing normal dispersion, using either passive dispersion-decreasing fiber or a hybrid configuration with Raman amplification [7]. The temporal and spectral profiles of the similariton generated in a fiber amplifier are independent of the input pulse profile; they are determined only by the input pulse energy and amplifier parameters [8]. The linear chirp of parabolic similariton is independent of the input pulse energy and depends only on the gain and dispersion of the amplifier [3]. Due to these unique properties, similaritons have interesting applications for the signal analysis-synthesis problems in ultrafast optics, particularly for the synthesis of optical pulses [9], for the pulse temporal compression [10], etc.
All the abovementioned studies are related to the parabolic pulse class of similaritons. Recently, a new type of similariton was generated in a passive fiber (without gain) under the combined effects of Kerr nonlinearity and dispersion [11, 12]. The applications of this similariton for temporal lensing / spectral compression, fine frequency tuning and femtosecond pulse imaging were also demonstrated $[13,14]$.

In this work, we study the generation of nonlinear-dispersive (NL-D) similariton of passive fiber, its distinctive properties, especially, its origin, nature and relation with the spectron and rectangular pulses, and the temporal, spectral and phase features in view of potential applications.

The outline of our work is the following: first, we carry out a rough analytical discussion and numerical studies to reveal the features of NL-D similariton, and afterwards we carry out experimentally the complete spectral interferometric characterization of NL-D similariton to check the terms of theory. Then we measure the similariton chirp by the use of spectrometer and autocorrelator, and finally, we study the bandwidth / duration rule of NL-D similariton. 


\section{ROUGH ANALYTICAL DISCUSSION}

First, we consider the pulse propagation through a pure $D$ medium. In the far field of dispersion, we have a spectron pulse [15], which repeats its spectral profile, in the temporal analogy of the Fraunhofer zone diffraction, and therefore propagates self-similarly. Mathematically, the solution of the second order dispersion equation in the temporal Fraunhofer zone $\left(z \gg L_{D}\right)$ obtains the form:

$$
A(z, t) \approx(-i \gamma)^{1 / 2} \exp \left(i \gamma t^{2} / 2\right) F T[A(0, t)]_{\omega=\gamma t} .
$$

In Eq. (1), $A(z, t)$ is the slowly varying complex temporal amplitude at the propagation distance $z, F T$ - the operator of Fourier transformation with $\omega=\gamma t$ and scaling coefficient equal to the chirp slope $\gamma \equiv d \omega / d t \approx-\left[\phi^{\prime \prime}\left(\omega_{0}\right)\right]^{-1}=$ $\left(\beta_{2} z\right)^{-1} ; \phi^{\prime \prime}\left(\omega_{0}\right)$ - second derivative of the $D$-induced spectral phase $\phi(\omega)$ at the central frequency $\omega_{0}, \beta_{2}$ - second order dispersion coefficient, $L_{D} \equiv\left(\beta_{2} \Delta \omega_{0}^{2}\right)^{-1}-D$ length, and $\Delta \omega_{0}$ - input bandwidth $[15,16]$. The condition of temporal Fraunhofer zone, meaning enough large pulse stretching $s \equiv \Delta t / \Delta t_{o} \approx \Delta \omega_{o}^{2} / \gamma \gg 1$, determines the $1 / s \approx \gamma / \Delta \omega_{o}^{2}$ precision of the spectron's spectrotemporal similarity $|A(z, t)| \propto$ $\left|F T[A(0, t)]_{\omega=\gamma t}\right|=\left|F T[A(z, t)]_{\omega=\gamma t}\right|$. For a 100 fs-pulse at the wavelength $\lambda=800 \mathrm{~nm}$ with the bandwidth $\Delta \lambda_{0} \sim 10 \mathrm{~nm}$, the $D$ length for standard single-mode fibers is $L_{D} \sim 10 \mathrm{~cm}$, and at the output of $1 \mathrm{~m}$-fiber we will have pulse stretching $s \approx z / L_{D} \sim 10$, and spectrotemporal similarity of spectron of the $1 / s \sim 10 \%$ precision.

For the NL-D propagating pulse, the NL self-interaction broadens the spectrum and increases the impact of dispersion, leading to higher precision spectron-similariton shaping. Quantitatively, for 100 fs-pulse radiation in a single-mode fiber with average power $p \sim 100 \mathrm{~mW}$ at a $76 \mathrm{MHz}$ repetition rate, the $N L$ interaction length $L_{N L} \equiv\left(\beta_{0} n_{2} I_{0}\right)^{-1}$ is much shorter than the $D$ one: $L_{N L} \sim 1 \mathrm{~cm} \ll L_{D} \sim 10 \mathrm{~cm}$. This allows us roughly splitting the impacts of $N L$ self-interaction and $D$ deformation of the pulse, assuming that first we have pure $N L$ self-phase modulation of the pulse and spectral broadening, and afterwards pure $D$ stretching and $D$-spectronic propagation. For the phase of output pulse, we have $\varphi_{D}(z, t)=\gamma t^{2} / 2$, and an additional phase term $\varphi_{N L}$ of $\left.F T\left[A\left(\sim L_{N L}, t\right)\right]\right|_{\omega=\gamma t}$, come from the pulse initial propagation step of $N L$ selfinteraction (at the distances $\sim L_{N L}$ ). Assuming it parabolic at the central energy-carrying part of the pulse at the propagation distances of $\sim L_{N L}$, we have the phase $\varphi_{N L}(z, t)=\gamma_{N L}^{-1} \omega^{2} /\left.2\right|_{\omega=\gamma t}=\gamma_{N L}^{-1}(\gamma t)^{2} / 2=\left(\gamma^{2} / \gamma_{N L}\right) t^{2} / 2$, with $\gamma_{N L} \equiv \varphi_{N L}{ }^{\prime \prime}\left(\sim L_{N L}, t\right)$. For the overall output phase $\varphi_{\Sigma}=\varphi_{D}+\varphi_{N L}$, we have $\varphi_{\Sigma}(z, t)=\gamma t^{2}\left(1+\gamma / \gamma_{N L}\right) / 2$. Considering the $N L$-spectral broadening and $D$-pulse stretching factors $\left(b \equiv \Delta \omega / \Delta \omega_{0}\right.$ and $\left.s \equiv \Delta t / \Delta t_{0}\right)$, we have for the $N L, D$, and overall chirp slopes at the output: $\gamma_{N L}=\Delta \omega / \Delta t_{0}=\Delta \omega_{0}^{2} b, \gamma=\Delta \omega_{0} / \Delta t=\Delta \omega_{0}^{2} / s$, and $\gamma_{\Sigma}=\gamma\left(1+\gamma / \gamma_{N L}\right)=\gamma\left[1+(s b)^{-1}\right]$. Since $\gamma / \gamma_{N L}=(s b)^{-1}$, for spectral broadening of $b \sim 10$ and pulse stretching of $s \sim 10\left(\Delta t_{0} \sim 100 \mathrm{fs}, p \sim 100 \mathrm{~mW}\right.$ average power at a $76 \mathrm{MHz}$ repetition rate, $z \sim 1 \mathrm{~m}$ of fiber), we will have $\gamma_{\Sigma}=\gamma\left(1+\gamma / \gamma_{N L}\right) \approx \gamma$, with the accuracy of $\gamma / \gamma_{N L} \sim 1 \%$.

Thus, for the femtosecond pulse NL-D self-interaction at $z \sim$
$1 \mathrm{~m}$ of fiber, we have spectron of $\sim 1 / \mathrm{s} b=\gamma / \Delta \omega^{2} \sim 1 \%$ precision. Considering the key peculiarity of the NL-D spectronsimilariton, that only the fiber dispersion determines the chirp slope, we can describe it following way:

$$
A(z, t) \approx(-i \gamma)^{1 / 2} \exp \left(i \gamma t^{2} / 2\right)\left|F T[A(z, t)]_{\omega=\gamma t}\right| .
$$

Another interesting issue is the relation of NL-D similariton with the rectangular pulses, shaped due to the pulse NL-D self-interaction at the fiber lengths $z \sim 2 \sqrt{L_{D} L_{N L}}[15,16]$. For such NL-D rectangular pulses the temporal stretching and spectral broadening are up to $\Delta t \approx 2 \Delta t_{0}$ and $\Delta \omega \approx$ $2 \Delta \omega_{0}\left(L_{D} / z\right)$, respectively, since the pulse optimal compression ratio is $\Delta t_{0} / \Delta t_{c} \approx \sqrt{L_{D} / L_{N L}} / 2=L_{D} / z[15,16]$, and $\Delta t_{0} / \Delta t_{c} \approx 2 \Delta \omega / \Delta \omega_{0}$. Thus, in this case the chirp slope obtains the value $\gamma=\Delta \omega / \Delta t \approx\left(2 \Delta \omega_{0} L_{D} / z\right) /\left(2 \Delta t_{0}\right)=$ $\left(\beta_{2} z\right)^{-1}$. Therefore, during the pulse NL-D self-interaction in fiber, the chirp slope becomes equal to the one of pulse $D$ propagation $\gamma \approx\left(\beta_{2} z\right)^{-1}$, starting from the fiber lengths $z \sim 2 \sqrt{L_{D} L_{N L}}$, and NL-D rectangular pulses can be considered as an earlier step of NL-D similariton shaping.

Summarizing the above analytical discussion, we can expect:

- a NL-D similariton of the spectron nature in the passive fiber;

- spectrotemporal similarity and imaging of the accuracy $\sim 1 / s b=\gamma / \Delta \omega^{2}$

- scaling coefficient of the chirp slope $\gamma \approx\left(\beta_{2} z\right)^{-1}$ for spectrotemporal imaging, determined only by the $D$ peculiarities of medium, and independent of the NL ones.

\section{SIMULATION}

In the mathematical description of the pulse NL-D selfinteraction in fiber, we use the standard nonlinear Schrödinger equation (NLSE) with the terms of Kerr nonlinearity and second order dispersion (group velocity dispersion), adequate to the pulse durations of $\geq 50$ fs $[15,16]$. We use the split-step Fourier method to solve NLSE. In simulations, the pulse propagation distance is expressed in $D$ lengths $L_{D}\left(L_{D} \sim 10 \mathrm{~cm}\right.$ for $100 \mathrm{fs}$ input pulses); the power of radiation in fiber is given by the $N L$ parameter $R \equiv L_{D} / L_{N L}=\left(\beta_{2} \Delta \omega_{0}^{2}\right)^{-1} \beta_{0} n_{2} I_{0} \sim I_{0}$ [15] ( $p=100 \mathrm{~mW}$ average power of a $100 \mathrm{fs}$-pulse radiation at a $76 \mathrm{MHz}$ repetition rate in a standard single-mode fiber corresponds to $R=6 ; p \leq 100 \mu \mathrm{W}$ is adequate to the pulse pure $D$ propagation of $R=0$ ). The dimensionless running time $t$ and centralized frequency $\Omega$ are normalized to the input pulse duration $\Delta t_{0}$ and bandwidth $\Delta_{\omega_{0}}=1 / \Delta_{t_{0}}$, respectively.

Figures 1 and 2 show the dynamics of similariton shaping: pulse (top row), chirp (middle row) and spectrum (bottom row) are shown during the pulse propagation in fiber. Figure 1 illustrates the first step of NL-D self-interaction, when typically, rectangular pulses are formed, and Figure 2 shows the step of similariton shaping. The spectral broadening and corresponding decrease of the pulse peak power lead to the "activation" of dispersion, the pulse obtains a linear chirp 



FIG. 1 Shaping of rectangular pulses $(R=30)$. From left to right: dynamics of the pulse propagation in fiber for $z / L_{D}=0.1 ; 0.2 ; 0.3$; and 0.4 . From top to bottom: pulse, chirp, and spectrum.


FIG. 2 Shaping and dynamics of NL-D similariton in fiber $(R=30)$. From left to right: $z / L_{D}=1 ; 2 ; 3 ; 4$. From top to bottom: pulse, chirp, and spectrum.

(parabolic phase), and the self-spectrotemporal similarity of NL-D similariton takes place (Figure 2).

Figure 3 shows the dynamics of the temporal and spectral profiles of NL-D similariton shaped from the initial pulses with sub-structure. At the output of the fiber, the pulse and spectrum have practically the same forms due to the linear chirp induced. Even in case of pulses with complex initial forms the output pulse has nearly parabolic form at its central energycarrying part. The irregularities of the temporal / spectral profiles are forced out to the edges during the pulse NL-D self-interaction, and spectrum and intensity become more and more parabolic. However, $D$ stretching of the pulse decreases its intensity which finally minimizes the impact of $N L$ selfphase modulation. The shape of spectrum does not change any more and the further alteration of the pulse shape has a $D$ character only.

Figure 4, for the NL-D similariton at a given fiber length $\left(z=4 L_{D}\right)$ and different power values, shows that the output chirp slope is practically independent of the input pulse intensity, and depends only on the fiber length. The temporal (a) and spectral (c) profiles and chirp (b) of NL-D similariton for different values of power $(R=0 ; 5 ; 10 ; 15)$ are shown. The black curves are for pure $D$ propagation of the pulse $(R=0)$. The red curves correspond to $R=5$, blue - to $R=10$, and



FIG. 3 NL-D self-interaction of a pulse with sub-structure and similariton shaping: the pulse (left) and spectrum (right) dynamics. Time and frequency are normalized to the current values of the pulse duration and bandwidth, $R=40$.


FIG. 4 Pulse (a), chirp (b), and spectrum (c) of NL-D similariton with the different values of power $(R)$, at the given fiber length $z=4 L_{D} . R=0 ; 5 ; 10 ; 15$ for the black, red, blue and green curves, respectively. The input pulse is Gaussian and transform limited

green - to $R=15$. The increase of the input pulse power leads to the spectral broadening and temporal stretching of pulse, keeping the chirp coefficient unchanged: the chirp slope is the same in all cases, even in case of pulse $D$-propagation $(R=0)$. It allows extracting the full information on the NL-D similariton having the spectrum and fiber length. Simulations are also carried out for higher powers (up to $R=50$ ), and the result is the same: chirp slope is independent of the power.

We studied the chirp of NL-D similariton versus the chirp of input pulse: the chirp slope of NL-D similariton is practically constant, when the pulse intensity is high enough. Figure 5 shows $\alpha \equiv-\phi^{\prime \prime}(\omega) \approx \gamma^{-1}$ versus the initial $\alpha_{0}$ for the different values of radiation power $(R)$. In contrast to pulse D propagation with $\alpha\left(\alpha_{0}\right)=\alpha_{0}+\beta_{2} z(R=0)$, we have a practically constant $\alpha\left(\alpha_{0}\right) \approx \beta_{2} z$ for powerful similariton $(R \gg 1)$. This is fulfilled enough good for pulses with positive initial chirps $\left(\alpha_{0}>0\right)$. In case of negative initial chirps $\left(\alpha_{0}<0\right)$, selfphase modulation results in spectral compression (in contrast



FIG. 5 Chirp of NL-D similariton $(\gamma)$ in case of different powers $(R): \alpha \equiv-\phi^{\prime \prime}(\omega) \approx$ $\gamma^{-1}$ vs input $\alpha_{0} \approx \gamma_{0}^{-1}$. Fiber length $z=15 L_{D} ; R=0$ (black), $R=10$ (red), $R=20$ (green), $R=30$ (blue), $R=40$ (magenta). 
to spectral broadening for $\alpha_{0}>0$ ), and $\alpha$ decreases. Anyway, in case of enough high intensities $\alpha$ becomes independent of the power and initial chirp slope $\alpha_{0}$. Figure 5 clearly shows the difference between the pulse pure $D$ (black line) and NL-D (colored curves) propagations in fiber. In case of the $D$ propagation the induced chirp simply imposes on the initial chirp. In case of NL-D propagation the chirp "forgets" about the initial chirp: it becomes independent of the input chirp.

\section{EXPERIMENT}

\subsection{Spectral interferometric study}

We carry out experimental studies to check-confirm the terms of our rough discussion of Section 2 and numerical analysis of Section 3. We use the spectral interferometric method of [17] to completely characterize and study the generation process and peculiarities of NL-D similariton of a passive fiber. Figure 6 schematically illustrates our experiment. Using a MachZender interferometer, we split the input radiation of a standard Coherent Verdi V10-Mira 900 F femtosecond laser system into two parts. The low-power pulse serves as a reference. For the high-power pulse, first we filter its spectrum of the bandwidth $\Delta \lambda=11 \mathrm{~nm}$ down to the value $\Delta \lambda=2 \mathrm{~nm}$.

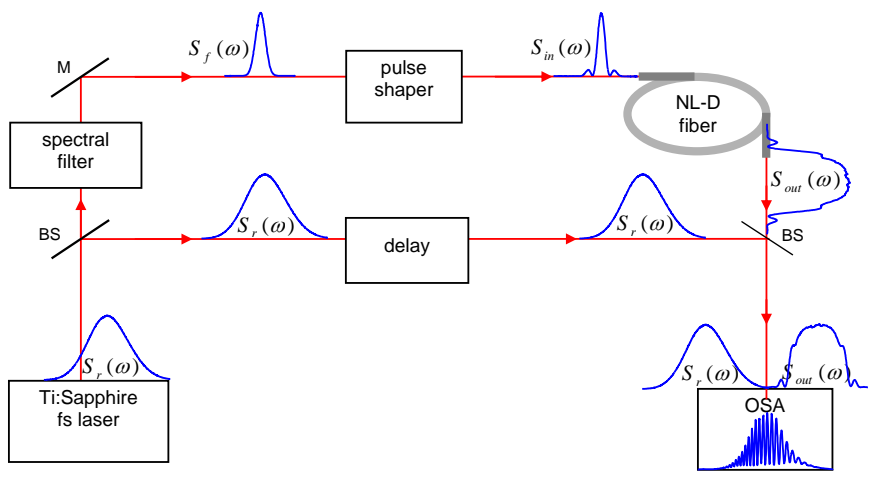

FIG. 6 Schematic of the experiment. M is mirror, BS - beam splitter, $S_{r}(\omega)$ - reference spectrum, $S_{f}(\omega)$ - spectrum filtered, $S_{i n}(\omega)$ - fiber input spectrum, $S_{\text {out }}(\omega)$ spectrum of NL-D similariton, OSA - optical spectrum analyser.

We use standard polarization-preserving fibers Newport FSPF @820 nm and ThorLabs HP @780 nm of different lengths: $1 \mathrm{~m}, 9 \mathrm{~m}$, and $36 \mathrm{~m}$. The spectra of the pulses at the output of the fiber are broadened, however the spectrum of the reference pulse covers them completely. This allows us measuring the spectral phase of similariton within the whole range of its spectrum. We record the spectral interferometric fringe pattern by an optical spectrum analyzer (OSA Ando 6315) and retrieve the spectral phase. Having the spectrum and retrieved spectral phase, we simulate the temporal profile of the similariton by Fourier transformation.

The performance of our experiment is given schematically in Figure 7 by means of the spectrograms of relevant steps. Figure 7(a) shows the spectrum of the laser pulse, (b) is the spectrum of spectrally filtered and shaped pulse, (c) is the spectrum of NL-D similariton and (d) is the spectral interferometric fringe pattern. Figure 7(e) shows the measured spectral phases of the similaritons generated from different input
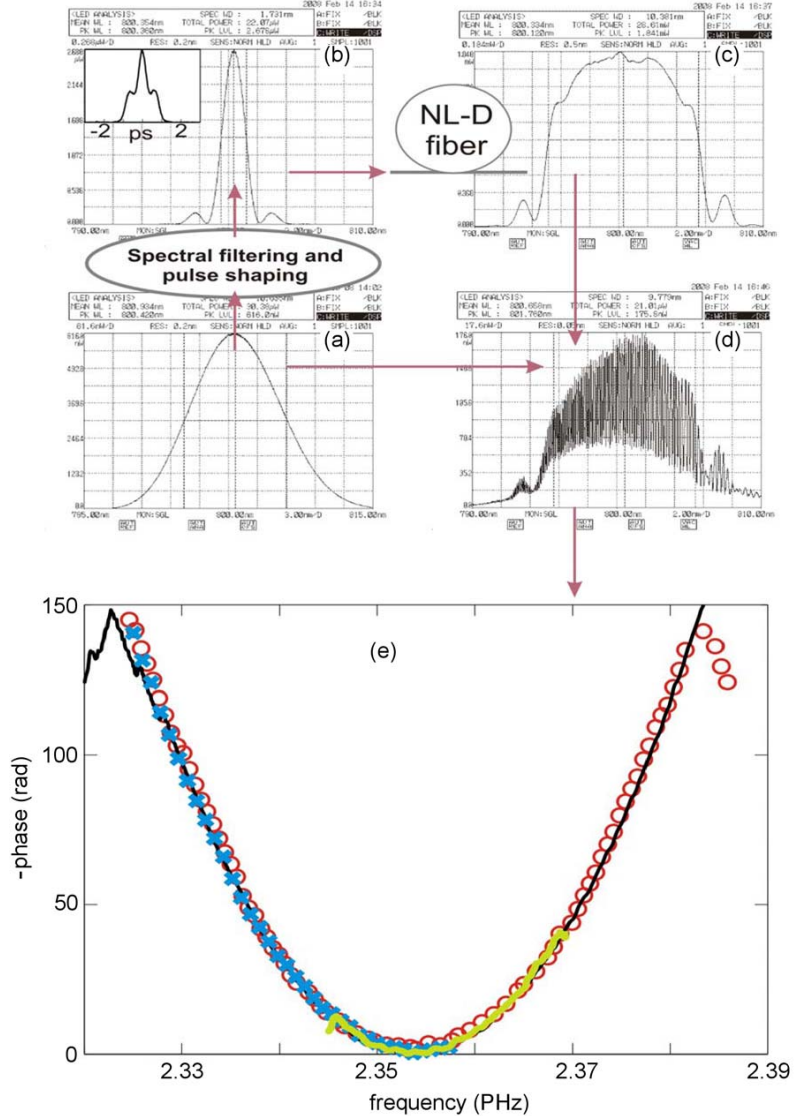

FIG. 7 (a-d) Schematic of the experiment given by the spectrograms of the relevant steps; (e) Spectral phases of NL-D similaritons generated from input single- (black), double- (red $\circ$ ) and distant double-peak (blue $\times$ ) pulses in comparison with the one for pure $D$ propagated single-peak pulse (yellow).

pulses. The spectral phases are parabolic $\left(\phi=-\alpha \omega^{2} / 2\right)$ and their coefficients $\alpha$ have nearly the same values in all cases of $D$ and NL-D propagations: $\alpha=0.32 \mathrm{ps}^{2}$ for the pure $D$ propagation of single-peak pulse, and $0.33 \mathrm{ps}^{2}, 0.328 \mathrm{ps}^{2}, 0.35 \mathrm{ps}^{2}$ for the NL-D propagations of single-, double- and distant doublepeak pulses, respectively. The parabolic phase (linear chirp) leads to the self-spectrotemporal imaging of similariton. The accuracy of imaging increases with the decreasing of the chirp slope, which is approximately equal to $\gamma \approx \alpha^{-1}$. Figures 7 and 8 illustrate the typical behaviour of NL-D similariton in case of $z=9 \mathrm{~m}$.

Having the spectral phase and spectral profile, we retrieve the temporal profile of NL-D similariton. Figure 8 shows the spectral and temporal profiles of the similaritons with the spectral phases of Figure 7(e). The black curves are the spectra and the gray-dotted curves are the pulses. They coincide with each other, that is, takes place self-spectrotemporal imaging of NLD similariton. A good spectrotemporal similarity is seen in case of input single-peak pulse (a). For input double- and distant double-peak pulses of (b) and (c) the matching between the spectral and temporal profiles of similaritons is qualitative only. To obtain a quantitative agreement, one must use a longer fiber, increasing the $\alpha$ coefficient: the spectral and temporal profiles of similaritons practically coincide for the orange lines of (b) and (c), showing the similariton temporal profiles for the increased $\alpha$ coefficients. 



FIG. 8 Self-imaging of NL-D similaritons generated from input single- (a), double- (b) and distant double-peak (c) pulses. Black solid curves show the spectra of NL-D propagated pulses (the black dotted one of (a) stands for pure $D$ propagated pulse), gray dotted lines show the retrieved temporal profiles. Orange curves of (b), (c) show the temporal profiles of similaritons for $4 \alpha$ increased coefficient of spectral phase.

To show the relation between the NL-D similariton and the rectangular pulse of fiber, we carry out spectral interferometric measurements using a short fiber $(z=1)$. Figure 9 illustrates the shaping of a rectangular pulse in NL-D fiber. Here the black curves are the spectrum and spectral phase measured, the blue curve is a fitted high-order polynomial and the pink is a fitted parabola. The measured spectral phase has a parabolic shape only at the central energy-carrying part of the spectrum. Deviation from parabola at the wings leads to the shaping of a rectangular pulse shown in Figure 9(b) with the blue curve. Even in this case the chirp slope in the central energy-carrying part of the pulse/spectrum is also determined only by the fiber length $\left(\alpha=0.0465 \mathrm{ps}^{2}\right)$. The pink curve in Figure 9(b) is the retrieved pulse by the fitted parabolic spectral phase.
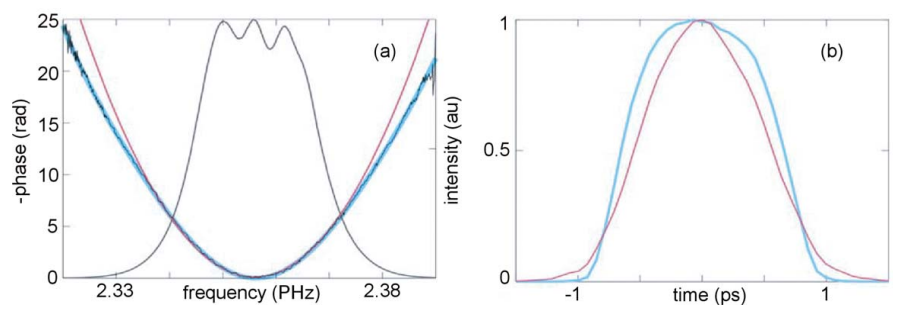

FIG. 9 (a) Spectrum of NL-D rectangular pulse with the relevant spectral phase (black solid line) and fitted parabola (pink). Blue line is the fitted high-order polynomial. (b) Temporal profile of the rectangular pulse (blue) in comparison with the pulse retrieved by the fitted parabolic spectral phase (pink).

Then we carry out experiment with a long fiber. This is the abovementioned regime of Figure 8 of high-value $\alpha$ coefficient, when accurate spectrotemporal similarity and imaging occur. Figure 10 shows the results of the experiment with $z=36 \mathrm{~m}$ fiber and the same input double-peak pulse as for Figure $8(\mathrm{~b})$. We simply show the spectra of NL-D similariton as its temporal images. In this regime, we have more accurate self-imaging of NL-D similariton, as compared with the regime of $z=9 \mathrm{~m}$ fiber (Figure 8). For comparison, the rel-


FIG. 10 Spectrotemporal images of similariton formed in $z=36 \mathrm{~m}$ fiber (c-f) in comparison with the one for $z=9 \mathrm{~m}$ (b). The radiation power increases from (c) to (f). (a) input spectrum with the autocorrelation track in the corner (autocorrelation duration at FWHM is $\tau=625 \mathrm{fs}$ )

evant spectral profiles of NL-D similaritons at the outputs of $9 \mathrm{~m}$ and $36 \mathrm{~m}$ fibers are shown together (Figures 10(b) and (e)).

\subsection{Chirp measurement for similariton by the use of spectrometer and autocorrelator.}

The precise spectral interferometric study of Section 4.1 confirmed the principal thesis of NL-D similariton description by Eq. (2), leading to its self-spectrotemporal similarity and imaging by the scaling coefficient of the chirp slope $\gamma=$ $\left(\beta_{2} z\right)^{-1}$. This allows us carrying out the similariton chirp studies by a simpler way. We only measure the spectral profile (Figure 11(a)) and autocorrelation track (black curve, Figure 11(b)) of the similariton. Afterwards, we calculate the autocorrelation of the spectrum (blue curve, Figure 11(b)). The comparison of the measured and calculated autocorrelations extracts the chirp slope. We have good accordance between the chirp slope values measured by this and the spectral interferometric methods of Section 4.1.

Figure 12 illustrates the results of this experiment for 2 different fiber lengths $z=33 \mathrm{~cm}$ (a) and $z=95 \mathrm{~cm}$ (b): simi-


FIC. 11 Determination of the chirp slope of NL-D similariton. (a) spectrum of NL-D similariton, (b) measured autocorrelation track (black) and calculated autocorrelation of spectrotemporal image (blue). 

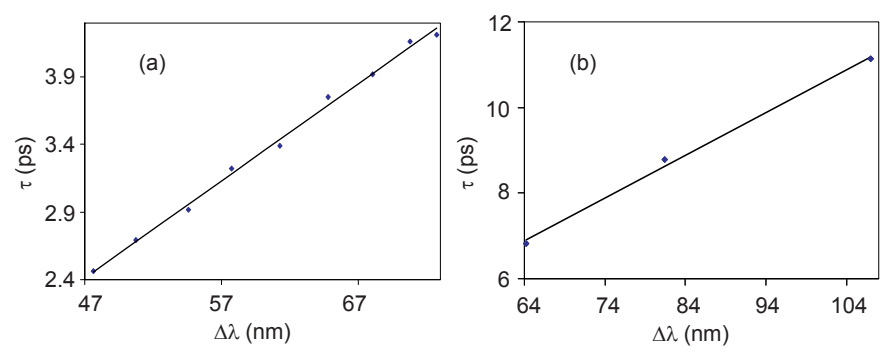

FIG. 12 Similariton's autocorrelation duration $\tau$ vs. bandwidth $\Delta \lambda$ for the fiber lengths $z=33 \mathrm{~cm}(\mathrm{a})$ and $z=95 \mathrm{~cm}(\mathrm{~b})$.

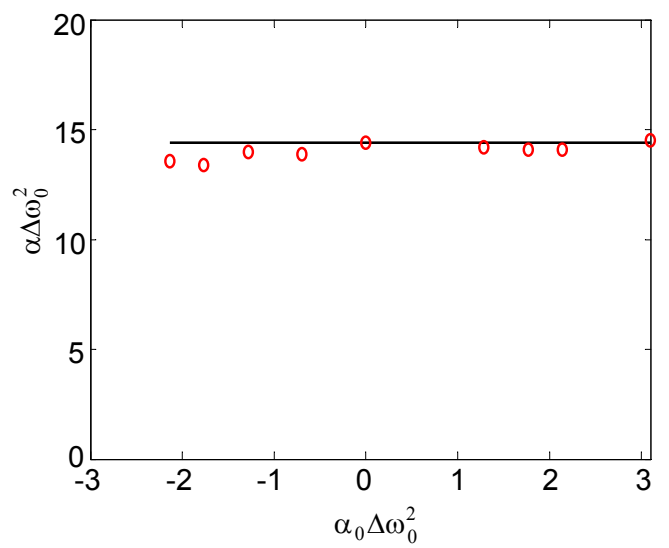

FIG. 13 Measured second derivative of spectral phase $\alpha$ of spectral phase of NL-D similariton vs. the one of the input pulse for the fiber length $z=14.4 L_{D}$. Red circles are the measured points, and the black line stands for $\alpha=\beta_{2} z$.

lariton's autocorrelation duration $\tau$ versus bandwidth $\Delta \lambda$ is shown. Actually, this curve gives the chirp of NL-D similariton. The chirp slope $\gamma=\alpha^{-1}$ extracted from the comparison of the measured and calculated autocorrelations is constant and it is independent of the bandwidth $\Delta_{\lambda}$ ( $\sim$ input power). In case of the fiber length $z=33 \mathrm{~cm} \alpha_{\lambda}=44.22 \mathrm{fs} / \mathrm{nm}$ which corresponds to $\alpha=0.015 \mathrm{ps}^{2}$. For $z=95 \mathrm{~cm} \alpha_{\lambda}=112 \mathrm{fs} / \mathrm{nm}$ which corresponds to $\alpha=0.038 \mathrm{ps}^{2}$, in good agreement with the spectral interferometric measurements of Section 4.1

This simple method permits checking easily the results on similariton chirp numerical study of Figure 5. Figure 13 shows the experimental results for the second derivative $\alpha$ of spectral phase of NL-D similariton versus the one of the input pulse for the fiber length $z=14.4 L_{D}$. One can see good agreement between the experiment (Figure 13) and simulation (Figure 5). Both curves show that the chirp slope of NL-D similariton is practically independent of the input pulse phase modulation in a wide range of $\alpha_{0} \Delta \omega_{0}^{2}$ from -3 to +3 .

\subsection{Bandwidth / duration of NL-D similariton}

Taking into account the relation between NL-D similariton and rectangular pulse revealed in Sections 2 and 4.1, it seems reasonable to expect that the bandwidth of similariton is equal to one for rectangular pulse. To determine the bandwidth (and afterwards the duration) of NL-D similariton, we can use the relation for the pulse optimal compression $[15,16]$. This gives the following relation for the spectral broadening of NL-D similariton: $b \equiv \Delta \omega / \Delta \omega_{0} \approx \sqrt{R} \equiv \sqrt{L_{D} / L_{N L}}=$
$C \sqrt{W / \Delta t_{\text {in }}} / \Delta \omega_{0}=C \sqrt{P} / \Delta \omega_{0}$, where $P$ is the input pulse power, $\Delta t_{\text {in }}$ - input pulse duration, $W=p / v$ - pulse energy, $p$ - average power of pulse radiation at a repetition rate $v$, and $C \equiv \sqrt{n_{2} \beta_{0}\left(\beta_{2} S\right)^{-1}}$ is a constant given by the fiber parameters $\left(n_{2}-\right.$ coefficient of the Kerr nonlinearity, $\beta_{0}=2 \pi / \lambda_{0}-$ wave number, $\beta_{2}$ - group-velocity dispersion coefficient, $S-$ fiber mode area). We check this thesis (Figure 14) numerically (a) and experimentally (b).
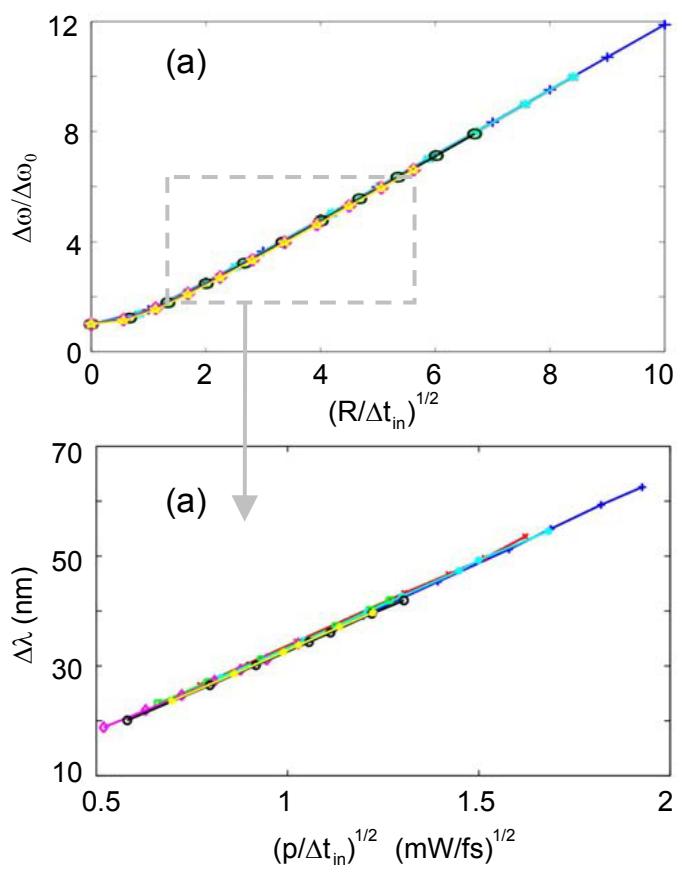

FIG. 14 (a) Simulation: spectral broadening versus $R$; (b) Experiment: similariton bandwidth $\Delta \lambda$ versus $\left(p / \Delta t_{i n}\right)^{1 / 2}$. Blue lines correspond to the transform-limited pulse of 100 fs duration, red and cyan - to 140 fs-pulse, green and black - to 225 fs-pulse, magenta and yellow - to 320 fs-pulse. Red, green and magenta are related to pulses stretched in $+D$ medium, and cyan, black, and yellow - to pulses stretched in $-D$ medium.

The numerical and experimental results of Figure 14 substantiate on the truthfulness of the brief discussion above. Thus, our studies give the following rule for the $\Delta \omega$ bandwidth and $\Delta t$ duration of NL-D similariton:

$$
\Delta \omega=C \sqrt{P}, \quad \Delta t=\Delta \omega / \gamma=C \beta_{2} z \sqrt{P} .
$$

For comparison, the bandwidth of the similariton generated in a fiber amplifier is $\Delta \omega(z)=$ $\left[\left(g \beta_{0} n_{2} P W\right) /\left(2 \beta_{2}^{2} S\right)\right]^{1 / 3} \exp (g z / 3)$, where $W$ and $P$ are the input pulse energy and power, and $g$ is the gain coefficient [3].

This revealed property of NL-D similariton of Eq. (3) can be used for the measurement of femtosecond pulse duration alternatively to the autocorrelation technique.

\section{CONCLUSION}

By the use of spectral interferometry, we have demonstrated the following properties of NL-D similariton generated in a passive fiber: 
- the linear chirp, with a slope given only by the fiber dispersion and independent of the amplitude, chirp and power of the input pulse;

- the relation with the rectangular pulse of NL-D fiber;

- the property of spectrotemporal similarity / selfspectrotemporal imaging, with the accuracy determined by spectral broadening and pulse stretching together;

- only initial pulse power determines the bandwidth of similariton.

\section{ACKNOWLEDGEMENTS}

This work was supported by the North Atlantic Treaty Organization within the framework of the Science for Peace program (project 978027).

\section{References}

[1] J. M. Dudley, C. Finot, G. Millot, and D. J. Richardson, "Selfsimilarity and scaling phenomena in nonlinear ultrafast optics" Nat. Phys. 3, 597-603 (2007).

[2] D. Anderson, M. Desaix, M. Karlson, M. Lisak, and M. L. QuirogaTeixeiro, "Wave-breaking-free pulses in nonlinear optical fibers" J. Opt. Soc. Am. B. 10, 1185-1190 (1993).

[3] V. I. Kruglov, A. C. Peacock, J. D. Harvey, and J. M. Dudley, "Selfsimilar propagation of parabolic pulses in normal-dispersion fiber amplifiers" J. Opt. Soc. Am. B. 19, 461-469 (2002).

[4] C. Finot, G. Millot, C. Billet, and J. M. Dudley, "Experimental generation of parabolic pulses via Raman amplification in optical fiber" Opt. Express 11, 1547-1552 (2003).

[5] T. Hirooka and M. Nakazawa, "Parabolic pulse generation by use of a dispersion-decreasing fiber with normal group-velocity dispersion" Opt. Lett. 29, 498-500 (2004).

[6] F. Ö. Ilday, F. W. Wise, and F. X. Kärtner, "Possibility of self-similar pulse evolution in a Ti-sapphire Laser"0pt. Express 12, 2731-2738 (2004).

[7] C. Finot, B. Barviau, G. Millot, A. Guryanov, A. Sysoliatin, and S. Wabnitz, "Parabolic pulse generation with active or passive dispersion decreasing optical fibers" Opt. Express 15, 15824-15835 (2007).

[8] C. Finot, G. Millot, and J. M. Dudley, "Asymptotic characteristics of parabolic similariton pulses in optical fiber amplifiers" opt. Lett. 29, 2533-2535 (2004).

[9] C. Finot and G. Millot, "Synthesis of optical pulses by use of similaritons" Opt. Express 12, 5104-5109 (2004).

[10] V. I. Kruglov, D. Méchin, and J. D. Harvey, “High compression of similariton pulses under the influence of higher-order effects" J. Opt. Soc. Am. B. 24, 833-838 (2007).

[11] P. Kockaert, L. Mouradian, G. Yesayan, and Ph. Emplit, "Generation of similaritons in a nonlinear dispersive optical fibre without gain" Proc. Symposium IEEE / LEOS Benelux Chapter, 39-42 (2007).

[12] G. Yesayan, K. Palanjyan, T. Mansuryan, A. Zeytunyan, L. Mouradian, P. Kockaert, and P. Emplit, "Nonlinear-spectronic similariton of single-mode fiber without gain" in Nonlinear Photonics, OSA Technical Digest (CD), paper JWA18 (Optical Society of America, 2007).

[13] A. Zeytunyan, T. Mansuryan, M. Kalashyan, G. Yesayan, L. K. Mouradian, F. Louradour, and A. Barthélémy, "Spectrotemporal imaging by sum frequency generation: ultrafast optical oscilloscope" in Frontiers in Optics, OSA Technical Digest (CD), paper FThD3 (Optical Society of America, 2007).

[14] T. Mansuryan, A. Zeytunyan, M. Kalashyan, G. Yesayan, L. Mouradian, F. Louradour, and A. Barthélémy, "Parabolic temporal lensing and spectrotemporal imaging: a femtosecond optical oscilloscope" J. Opt. Soc. Am. B. 25, A101-A110, (2008).

[15] S. A. Akhmanov, V. A. Vysloukh, and A. S. Chirkin, Optics of femtosecond laser pulses (AIP, New York, 1992).

[16] G. P. Agrawal, Nonlinear Fiber Optics (4th edition, Academic, 2007).

[17] F. Reynaud, F. Salin, and A. Barthélémy, "Measurement of phase shifts introduced by nonlinear optical phenomena on subpicosecond pulses" Opt. Lett. 14, 275-277 (1989). 